\title{
QTL Mapping of Cold Tolerance at the Seedling Stage using Introgression Lines Derived from an Intersubspecific Cross in Rice
}

\author{
In-Kyu Park ${ }^{1}$, Chang-Sik $\mathrm{Oh}^{2}$, Dong-Min Kim ${ }^{1}$, Sang-Min Yeo ${ }^{1}$, and Sang-Nag $\mathrm{Ahn}^{1}{ }^{1}$. \\ ${ }^{1}$ Department of Agronomy, College of Agriculture \& Life Sciences, Chungnam National University, Daejeon 305-764, Korea \\ ${ }^{2}$ Present address: Biotech Research Center, Dongbu farm Hannong, Anseong-si, Korea
}

\begin{abstract}
Low-temperature stress is an important factor controlling the growth and development of rice (Oryza sativa L.) in temperate region. In this study, a molecular linkage map consisting of 136 SSR markers was employed to identify QTL associated with cold tolerance at the seedling stage. 80 recombinant inbred lines (RILs) from an intersubspecific cross between Milyang23 (O. sativa ssp. Indica) and Hapcheonaengmi3, a japonica weedy rice and the parents were evaluated for leaf discoloration and SAPD value of seedlings. Rice plants were grown for 15 days in the low-temperature condition $\left(13 / 20^{\circ} \mathrm{C}\right.$ day/night $)$ and the control condition $\left(25 / 20^{\circ} \mathrm{C}\right.$ day/night) in the growth chamber. The degree of leaf discoloration showed a highly significant correlation with the SPAD value in the low-temperature plot $(r=-0.708, P<0.0001)$. A total of four QTLs for SPAD were identified and the phenotypic variance explained by each QTL ranged from 5.4 to $16.0 \%$. Two QTLs detected in the control condition were located on chromosomes 2 and 5 , respectively. Two QTL on chromosomes 1 and 4 were detected at the low-temperature condition and Hapcheonaengmi3 alleles increased the SPAD values at these loci. Substitution mapping was conducted to delimit the position of $q S P A-4$ using introgression lines derived from the same cross. Results indicated that $q S P A-4$ was located in a $810-\mathrm{Kb}$ region flanked by RM16333 and RM16368. The results indicated that Hapcheonaengmi3 contains QTL alleles that are likely to improve cold tolerance of Indica rice.
\end{abstract}

Keywords Rice, QTL, Cold tolerance, Seedling stage, SPAD value, Discoloration

\section{INTRODUCTION}

Low-temperature stress is a major factor limiting rice production in temperate and high altitude areas (IRRI 1979, Sthapit and Witcombe 1998). Rice can be sensitive to low-temperature, particularly during early seedling development, and cool temperature (below $15-17^{\circ} \mathrm{C}$ ) can cause severe injuries leading to poor establishment, decrease in the plant competitive ability against weeds, delayed crop maturation and subsequently decrease in yields (Yoshida et al. 1996; Andaya and Makill 2003). Rice plants are injured at the seedling stage when they are grown in early spring in temperate or subtropical environments. Especially, in the case of direct seedling cultivation in the low-temperature areas of Korea, germination and early growth of seedlings take place under relatively low-temperature conditions (Suh et al. 2003).

A wide range of variation in cold tolerance at various stages has been observed in rice and improving cold tolerance is one of the most important breeding objectives in Korea, particularly for Tongil-type rice. Tongil-type rice is more sensitive to cold than Japonica rice (Jeong et al. 2000). Depending on the severity and length of exposure of low temperature, the Tongil-types display more severe leaf yellowing or discoloration (chlorosis), seedling stunting of seedling, heading delay, spikelet sterility and poor panicle exsertion compared to the Japonica subspecies in the low temperature condition (Jeong et al. 2000).

With the development of diverse DNA markers and the construction of high density linkage maps for many plant

Received Feb 5, 2013; Revised Feb 22, 2013; Accepted Feb 27, 2013; Published March 31, 2013

*Corresponding author Sang-Nag Ahn, ahnsn@cnu.ac.kr, Tel: +82-42-821-5728, Fax: +82-42-822-2631 
species like rice, QTL (quantitative trait loci) analysis has become a powerful tool for the genetic dissection of quantitative traits (McCouch and Doerge 1995). Several QTL controlling cold tolerance at various growth stages have been identified (Misawa et al. 2000; Andaya and Mackill 2003; Saito et al. 2004; Suh et al. 2010). Genetic analysis of rice seedling cold tolerance has uncovered a large number of loci (Misawa et al. 2000; Zhang et al. 2005; Andaya and Tai 2006, 2007; Ji et al. 2010).

The appropriate screening procedure in evaluating low-temperature sensitivity in rice is an important issue. Leaf discoloration as a visual manifestation of cold damage in the growth chamber, greenhouse or in the cold water irrigation has been considered a reliable method in the breeding programs (Andaya and Mackill 2003; Oh et al. 2004; Andaya and Tai 2007). However, the visual scoring of discoloration is sometimes difficult when the symptoms are not pronounced in the segregating population. It also took a longer duration of stress before the symptoms were manifested. These add to the complexity in data interpretation and it is necessary to minimize these problems.

Jeoung et al. (2002) examined the mechanism of chilling on photosynthesis using two rice accessions. When the leaves were exposed to various low temperatures, the leaf photosynthesis of Milyang2 3 decreased faster than that of Stejaree45 indicating that Stejaree45 (temperate Japonica) was more resistant to light-chilling stress than Milyang23 (Indica). Because there is a positive relationship between leaf photosynthesis and leaf chlorophyll content (KuraHotta et al. 1987; Kim et al. 2002), it should be possible to use chlorophyll content as a manifestation of cold damage. A method to measure leaf chlorophyll content is widely employed using a digital chlorophyll meter, and a positive correlation between Soil and Plant Analyzer Development (SPAD) values and leaf photosynthesis values have been observed in rice (Watanabe et al. 1980; Kim et al. 2002; Takai et al. 2010).

In this study, we focused on the SPAD value and yellowing of rice seedlings to detect and fine map QTLs for cold tolerance using recombinant inbred lines (RILs) and introgression lines (ILs) derived from an intersubspecific cross between Hapcheonaengmi3 and Milyang23.

\section{MATERIALS AND METHODS}

\section{Plant materials and mapping populations}

Milyang23 (Oryza sativa ssp. Indica) was used as the female parent and also served as the recurrent parent. Hapcheonaengmi3, a japonica weedy rice, is photoperiod insensitive, and has a higher level of cold tolerance compared to Korean japonica rice accessions (Suh et al. 2003). 80 recombinant inbred lines from a cross between MIlyang23 and Hapcheonaengmi3 were used for QTL analysis (Oh et al. 2004) and introgression lines derived from the same cross were used for substitution mapping of QTL (Oh et al. 2010).

\section{Evaluation of cold tolerance}

Evaluation of cold tolerance was done in the growth chambers (MLR-351H, SANYO co., Japan and HB-303 DH-L, Vision Co., Korea). The light was provided for $14 \mathrm{~h}$ period inside the growth chamber. The light intensity was about 20,000 lux while the relative humidity ranged from $70-75 \%$. Two chambers were used for the experiment; one chamber for the control plot and the other for the treatment plot. Control plot condition was $25^{\circ} \mathrm{C}$ day $/ 20^{\circ} \mathrm{C}$ night and low temperature was $13^{\circ} \mathrm{C}$ day $/ 20^{\circ} \mathrm{C}$ night. The experiment was conducted in a completely randomized design with two replications. Ten seeds of each RIL were planted in rice nursery bed (Punong Co., Korea). Seeds were allowed to germinate and grow until three-leaf stage in the growth chamber set at $25 / 20^{\circ} \mathrm{C}$, for $14 \mathrm{~h}$ photoperiod. 15 days after seeding, a growth chamber was adjusted to low-temperature regime. After low-temperature regime treatment for 15 days, we evaluated yellowing and SPAD value of each plot. Phenotypic scoring based on yellowing was performed at 15 days of treatment using the scale of one (green, normal growth) to nine (yellow, stunted) as described previously (Andaya and Mackill 2003). SPAD values of seedlings were measured with a SPAD meter (SPAD-502, KonicaMinolta, Japan). At three-leaf stage, SPAD values were obtained from the middle of leaf blade in the third-leaf from 10 plants for each RIL. The SPAD scores were averaged and used in the analysis. 


\section{DNA extraction and Simple Sequence Repeats analysis}

DNA was extracted from the fresh leaf tissues of each line in bulk according to the chloroform-based DNA extraction protocol (Causse et al. 1994). SSR analysis was performed according to the method described in Panaud et al. (1996). A total volume of $20 \mathrm{uL}$ reaction mixture was composed of the $5.0 \mathrm{uL}(5 \mathrm{ng} / \mathrm{uL})$ of template DNA, Taq polymerase $0.1 \mathrm{uL}$ (5 Unit/uL), $0.8 \mathrm{ul} \mathrm{dNTP}(2.5 \mathrm{mM}$ each), Forward + Reverse primer 1ul (10 pmol each), $2.0 \mathrm{ul}$ 10 x PCR buffer (10 mM Tris-HCl PH 8.3, 50 mM KCl, 1.5 $\mathrm{mM} \mathrm{MgCl}_{2}, 0.1 \%$ Gelatin), and 11.1 ul triple distilled water. Amplification was accomplished using BioRad Thermo Cycler according to the step-cycle program set for initial denaturing step with $94^{\circ} \mathrm{C}$ for $5 \mathrm{~min}$, followed by 35 cycles for $1 \mathrm{~min}$ at $94^{\circ} \mathrm{C}, 1 \mathrm{~min}$ at $55^{\circ} \mathrm{C}$ or $58^{\circ} \mathrm{C}, 2 \mathrm{~min}$ at $72^{\circ} \mathrm{C}$, with a final extension at $72^{\circ} \mathrm{C}$ for $5 \mathrm{~min}$. PCR products were separated on $4 \%$ polyacrylamide denaturing gel for 1-2 hours at 1800-2000V. SSR marker bands were revealed using silver staining kit of the Bioneer Co. (www.bioneer.co.kr). Primer sequences of published rice microsatellite (RM) makers located within the target region were obtained from Gramene data base (www.gramene.org, McCouch et al. 2002). For substitution mapping, additional SSR markers were identified from the Nipponbare genome sequence information (www.gramene.org).

\section{QTL analysis}

The location of QTLs was determined by single-point analysis (SPA) and composite interval mapping (CIM). Single point analysis (SPA) was performed to determine the effect of each marker on each trait. In SPA, a QTL was declared if the phenotype was associated with a marker locus at $P<0.001$ or with two adjacent marker loci at $P$ $<0.05$. QTL analysis was performed by composite interval mapping (CIM) using the QTL Cartographer version 2.5 software (Wang et al. 2007). Significant thresholds for CIM were determined using 1000 permutations. For CIM, the experiment-wise significance level of $P<0.01$ corresponded to a LOD score of 3.60, while the level of $P<0.05$ corresponded to an LOD $>2.51$. The QTL reported in this study were detected by both methods. The proportion of observed phenotypic variation attributable to a particular QTL was estimated by the coefficient of determination
$\left(\mathrm{R}^{2}\right)$. In the fine mapping of the position of $q S P A-1$ and $q S P A-4$, the substitution mapping strategy was used. In each IL, the mean phenotypic value was compared with that of the controls Milyang23 and Hapcheonaengmi3 using the SAS statistical software package (SAS 1990, SAS Institute, Cary, NC, USA) at a significant level of $P<$ 0.05 .

\section{RESULTS}

\section{Performance of the parents in cold-tolerance related traits}

Two parents were grown in the low temperature and the control plots. At the control plot, no difference between the parents was observed in the degree of seedling yellowing and in SPAD value (data not shown). Milyang23 and Hapcheonaengmi3 scored 1 for seedling yellowing in the control (data not shown). However, the parents showed difference in the degree of seedling yellowing at the low-temperature plot. Milyang23 and Hapcheonaengmi3 scored 9 and 1 for yellowing in the treatment plot, respectively (Fig. 1). Third leaf of Milyang23 showed severe yellowing than that of Hapcheonaengmi3. Milyang23 started to develop the symptom as early as 13 days of

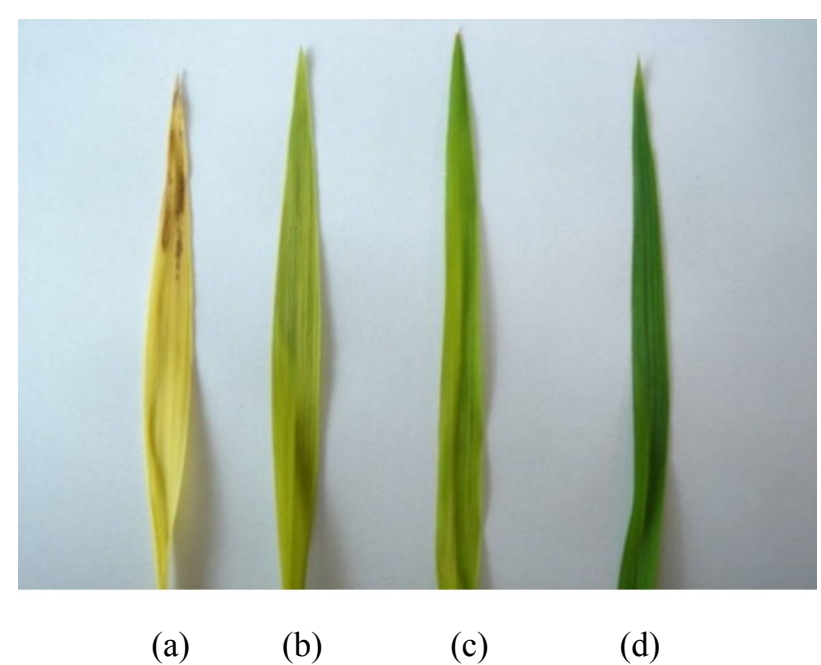

Fig. 1. Discoloration of the third leaves among RILs and the parents at the low temperature condition 15 days after treatment. (a) Milyang23, (b), (c) two RILs and (d) Hapcheonaengmi3 


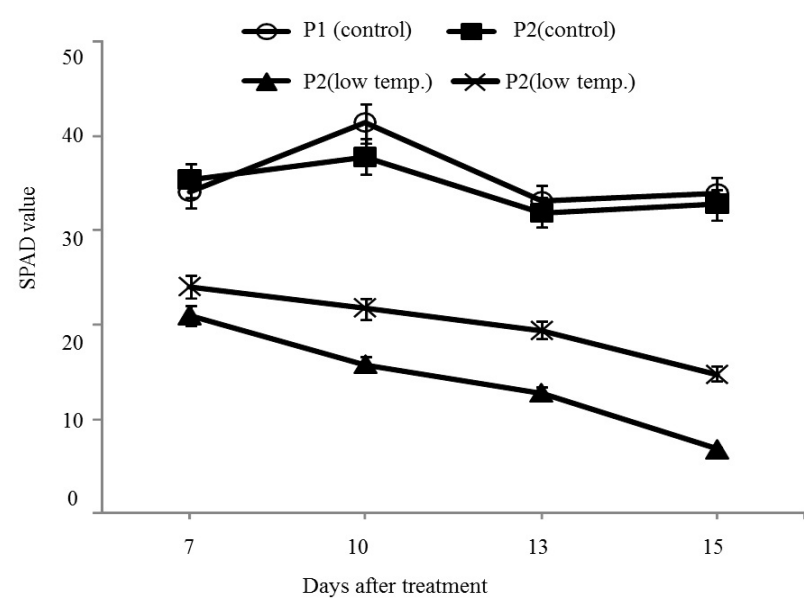

Fig. 2. Change in SPAD values in the parents according to days after low-temperature treatment. Vertical bars show the standard deviation (mean $\pm \mathrm{sd})$. $\left(\mathrm{P}_{1}\right.$ : Milyang23, $\mathrm{P}_{2}$ : Hapcheonaengmi3).

treatment whereas Hapcheonaengmi3 did not show leaf yellowing. SPAD values were measured for the seedlings of the parents and Fig. 2 compared SPAD values between the parents in the low-temperature treatment and the control plots. No difference in SPAD value between the parents was observed at the control plot. Also, no difference was seen among rice seedlings according to the growth duration in the control condition. However, difference in SPAD value between the parents was observed 7 days after treatment and thereafter in the low- temperature plot. At 15 days after treatment, the difference in SPAD value between the parents reached the maximum and 15-day treatment was chosen for evaluation of RILs for cold tolerance.

\section{Phenotypic variation of the RILs}

80 RILs were grown in the low-temperature plot and the control plot. RILs showed phenotypic variation in yellowing at the low-temperature (Fig. 1). Fig. 1 shows the third leaf of the parents and two lines. Frequency distribution of SPAD values in both plots for 80 RILs is shown in Fig. 3. SPAD value showed continuous and normal distributions. The mean SPAD value of Milyang23 was 33.9 in the control and 6.7 in the low temperature. The SPAD values for 80 RILs ranged from 25 to 50 in the control plot and from 15 to 25 in the low temperature. Difference in SPAD value of the RILs between the control plot and the low

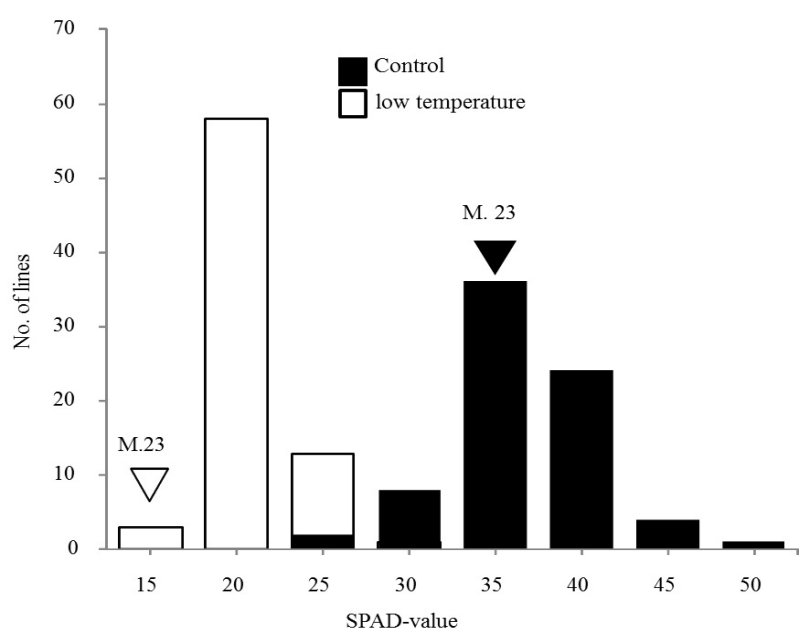

Fig. 3. Frequency distribution of SPAD values in the 80 RILs. Difference in SPAD values of the 80 RILs between the control and low-temperature plots was highly significant $(t=47.2, P<0.001)$. $\left(\mathrm{P}_{1}\right.$ : Milyang 23, $\mathrm{P}_{2}$ : Hapcheonaengmi3).

temperature plot was highly significant $\left(t=47.2^{* * *}\right.$, $P<0.0001)$.

\section{QTL analysis}

The degree of yellowing showed a highly significant correlation with the SPAD value in the RIL in the lowtemperature plot $(r=-0.708, P<0.0001)$ and the SPAD

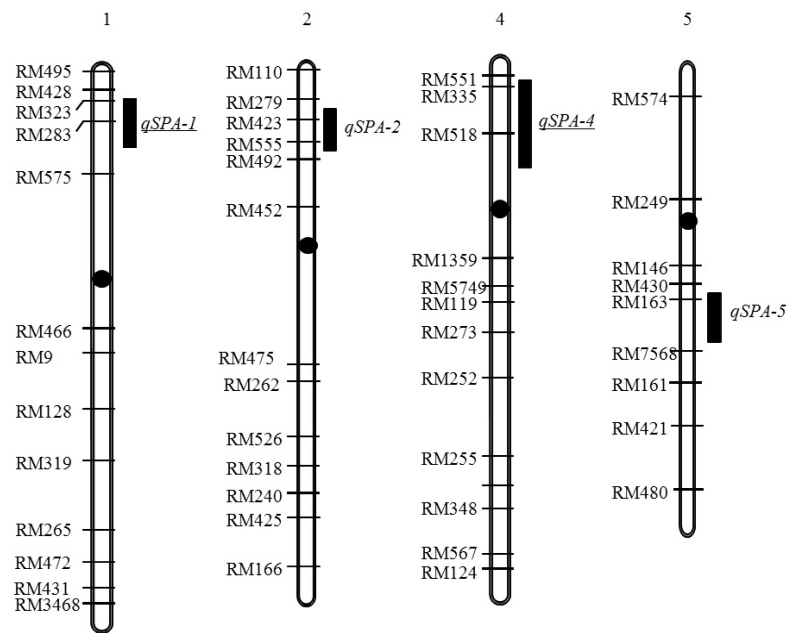

Fig. 4. Map locations of the putative QTLs associated with SPAD values. QTLs detected are shown on the right of chromosomes. QTLs detected in the control are underlined. 
Table 1. Characteristics of the QTL detected in the 80 RILs.

\begin{tabular}{|c|c|c|c|c|c|c|c|c|c|}
\hline \multirow[b]{2}{*}{ Trait } & \multirow[b]{2}{*}{ QTL } & \multirow[b]{2}{*}{ Chr. } & \multirow[b]{2}{*}{ Marker } & \multicolumn{3}{|c|}{ Control plot } & \multicolumn{3}{|c|}{ Low temperature plot } \\
\hline & & & & $\mathrm{P}$ & $\mathrm{R}^{2 \mathrm{z})}$ & $\begin{array}{c}\text { Increased } \\
\text { Effect }^{b)}\end{array}$ & $\mathrm{P}$ & $\mathrm{R}^{2 \mathrm{y})}$ & $\begin{array}{c}\text { Increased } \\
\text { Effect }^{\mathrm{b})}\end{array}$ \\
\hline \multirow{4}{*}{ SPAD value } & $q S P A-1$ & 1 & RM428-RM323 & - & - & - & 0.000 & 7.5 & $\mathrm{H}$ \\
\hline & $q S P A-2$ & 2 & RM423-RM555 & 0.01 & 11.1 & M & - & - & - \\
\hline & $q S P A-4$ & 4 & RM335-RM518 & - & - & - & 0.000 & 16.0 & $\mathrm{H}$ \\
\hline & $q S P A-5$ & 5 & RM163 & 0.01 & 5.4 & M & - & - & - \\
\hline
\end{tabular}

${ }^{\mathrm{z})}$ Coefficient of determination, ${ }^{\mathrm{y})} \mathrm{M}$ : Milyang23; H: Hapcheonaengmi3

values were used in the QTL analysis. Four significant QTLs were detected for SPAD values (Fig. 4, Table 1). Two QTLs were detected in the control plot near the markers RM423-RM555 and RM163 on chromosomes 2 and 5, respectively. At the $q S P A-2$ and $q S P A-5$ loci, Milyang23 alleles increased the SPAD values. The other two QTLs were detected in the low temperature plot near the marker interval of RM428-RM323 and RM335-RM518 on chromosomes 1 and 4, respectively. $q S P A-1$ and $q S P A-4$ accounted for 7.5 and $16.0 \%$ of the phenotypic variance in the low-temperature plot. At $q S P A-1$ and $q S P A-4$, Hapcheonaengmi3 alleles increased SPAD values. No interaction was observed between $q S P A-1$ and $q S P A-4$.

\section{Substitution mapping of qSPA-4}

$q S P A-4$ was mapped near SSR markers on the short arm of chromsome 4 (Fig. 4). To narrow down the position of $q S P A-4$, substitution mapping was conducted using

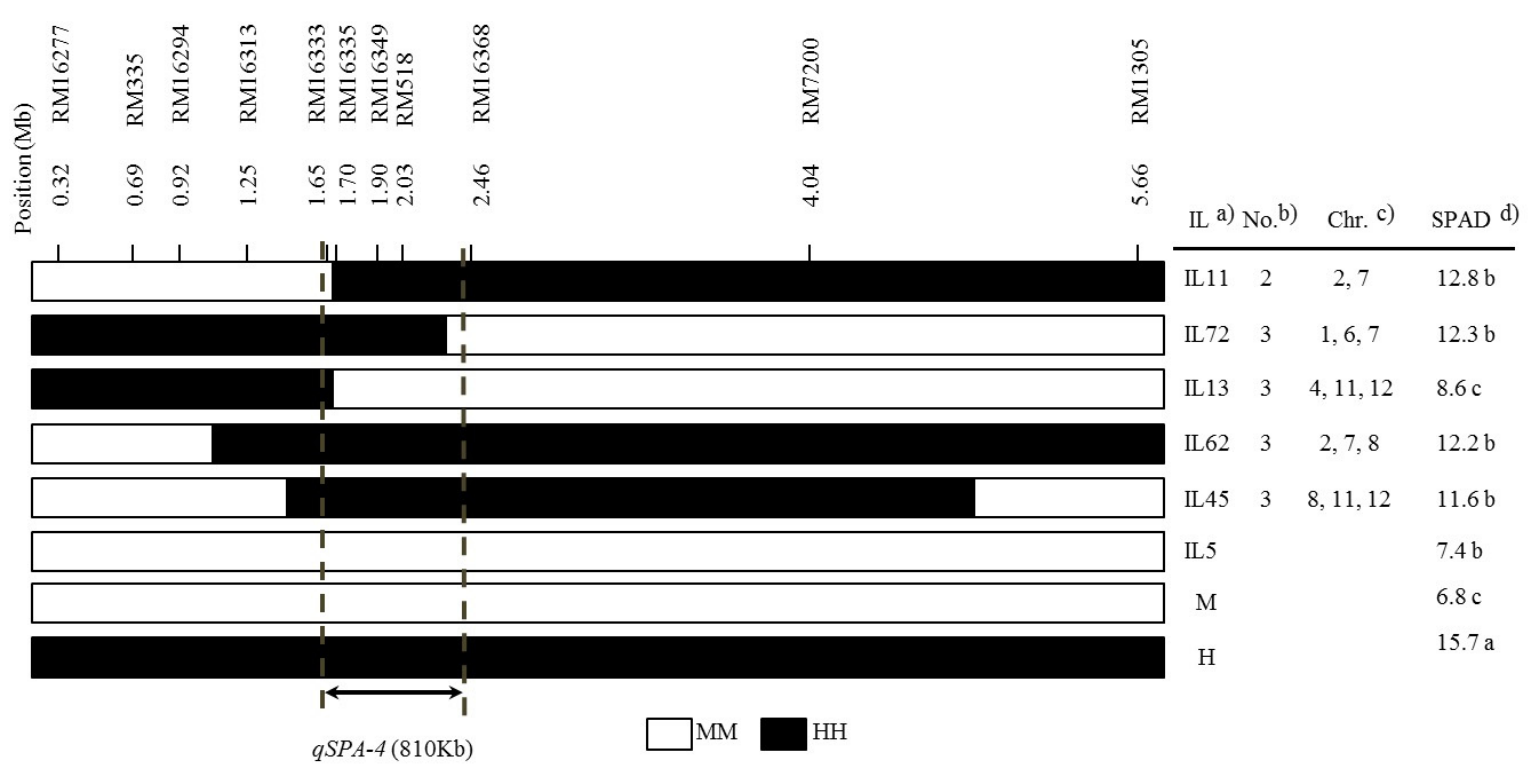

Fig. 5. Substitution mapping of the qSPA-4 QTL on the short arm of chromosome 4 based on 6 ILs, and two parents, Milyang23 and Hapcheonaengmi3. Black box denotes Hapcheonaengmi3 homozygous segment and white box denotes homozygous for Milyang23 alleles. Position of SSR markers is based on the Nipponbare genome sequence information (www.gramene.org). Chromosome region affecting SPAD value is shown by double-headed arrow on chromosome 4, predicted from the difference in SPAD value between each IL and Milyang23.

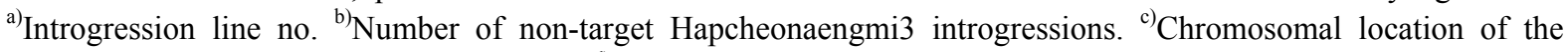
non-target Hapcheonaengmi3 introgressions. ${ }^{\mathrm{d})}$ Numbers followed by the same letters are not significant at a significant level of $p=0.05$ based on DMRT. 
introgression lines developed from the same cross as the present study (Oh et al. 2010). To fine map $q S P A-4,6$ ILs were selected (Fig. 5). The number of Hapcheonaengmi3 introgressions including the target region ranged from 3 (IL11) to 4 (IL13, IL45, IL62, and IL72). One IL, IL5 without the $q S P A-4$ was used as controls. These 6 lines did not have the $q S P A-1$ region on chromosome 1 . Selected ILs and the paterts were evaluated for tolerance to lowtemperature stress. Additional 7 SSR markers in the target regions were mapped. Two parents showed a significant difference in SPAD value. Among the 6 lines, IL13 was sinficantly lower in SPAD than Hapcheonaengmi3, sugesting that $q S P A-4$ was located in the downstream region of RM16333. IL11, IL72, and IL62 showed significantly higher SPAD values than Milyang23 indicating that $q S P A-4$ was located in an interval between RM16333 and RM16368 which are 810-kb apart on chromosome 4.

\section{DISCUSSION}

Cold tolerance of rice at the seedling stage is difficult to measure in a single test because of the complexity of injuries and symptoms caused by low-temperature stress. In this study, cold tolerance was evaluated based on the visual symptom and SPAD values observed during the low temperature treatment of the $13 / 20^{\circ} \mathrm{C}$ (day/night) regime. The seedlings treated under this condition suffered leaf yellowing as the duration of stress was prolonged. Milyang23, a susceptible Indica, started to develop the symptom as early as $13 \mathrm{~d}$ of treatment whereas Hapcheonaengmi3 did not show leaf discoloration. This finding is consistent with the result that Japonica was more resistant to cold stress than Indica (Jeoung et al. 2002; Zhang et al. 2005). SPAD values were also measured to provide a more accurate measurement than the visual score. The degree of leaf discoloration showed a highly significant correlation with the SPAD value in the RIL in the low-temperature plot $(r=-0.708, P<0.0001)$ and this finding supports the finding that low temperature affects the photosynthetic activity of leaves (Jeoung et al. 2002).

Takai et al. (2010) found that the mean SPAD value of flag leaves at heading stage was significantly higher in
Habataki (Indica) than in Sasanishiki (Japonica). However, the result of Abdelkhalik et al. (2005) indicated that the difference between Indica and Japonica in SPAD values at flowering and 25 days after flowering was not significant. In this study, the difference between the parents in the SPAD value was not statistically significant. These conflicting results might be attributed in part to the difference in sampling period (seedling stage and heading stage) and the parents that were selected for the study.

A number of studies discovered QTL for chlorophyll contents in rice (Ishimaru et al. 2001; Abdelkhalik et al. 2005; Takai et al. 2010). In this study, two QTLs, $q S P A-2$ and $q S P A-5$ for SPAD value were identified in the control plot and Milyang23 alleles increased the SPAD values at these loci. $q S P A-5$ detected in this study might correspond to the QTL for chlorophyll content near an RFLP marker R1553 previously reported by Ishimaru et al. (2001) using backcross inbred lines from a cross between Nipponbare and Kasalath. However, no previous study reported QTL for chlorophyll content near $q S P A-2$ on chromosome 2 suggesting that $q S P A-2$ is a new gene for chlorophyll content. Hapcheonaengmi3 performed better than Milyang23 at the low-temperature plot. Two QTLs, $q S P A-1$ and $q S P A-4$ were identified for SPAD value and Hapcheonaengmi3 alleles increased the SPAD values at these loci.

Few QTL for cold tolerance have been identified in a similar location as the $q S P A-1$ in this study (Andaya and Mackill 2003; Ji et al. 2010). Andaya and Mackill (2003) mapped a QTL, $q C T B 1$ for spikelet sterility between SSR markers, RM151 and RM259. qSPA-1 detected in this study was not at the same position as qCTB1. Recently, $q I W-1$ on chromosome 1 was determined to control plumule withering in the seedling stage in a DH population derived from a cross between $\mathrm{TN} 1$ and Chunjiang 06 (Ji et al. 2010). Likewise, the QTL detected in the present study appears to be different from $q I W-1$. Although the respective QTLs in these studies were not confirmed in nearly-isogenic backgrounds, $q S P A-1$ in this study might be not allelic to $q I W-1$ based on the position suggesting that $q S P A-1$ is a new gene for cold tolerance. The location of $q S P A-4$ was narrowed down to a $810-\mathrm{kb}$ interval between RM16333 and RM16368 on chromosome 4. qSPA-4 shared the similar location with $q C T S 4$ reported by Andaya and Tai 
(2007). qCTS4 is associated with tolerance to cold-induced yellowing and stunting from prolonged exposure to intermittent low-temperature and located in the $128-\mathrm{kb}$ region between CT245 and CT236. Additional experiment is needed to clarify whether $q S P A-4$ is allelic to $q C T S 4$.

We have identified the genetic loci associated with cold tolerance in a cross between the two main subspecies that represent tolerant (Japonica) and susceptible (Indica) rice germplasm. To our knowledge, this is the first QTLs to be identified controlling cold tolerance at the seedling stage using SPAD values obtained from RILs and ILs treated in the growth chamber. This study indicated that Hapcheonaengmi3 (Japonoca) contained QTL alleles that are likely to improve cold tolerance at seedling stage in Milyang23 (Indica). This result is consistent with previous reports that weedy rice is an important resource of tolerance because it possesses useful genes for tolerance to various biotic and abiotic stresses for a wide range of adverse conditions (Suh et al. 1997). The RIL and IL populations we developed from an Indica Milyang23 and a temperate Japonica weedy rice Hapcheonaengmi3, should be useful in examining important traits in both tropical and temperate environments. Weedy rice could be used as a good material of natural genetic variation underlying the evolution and breeding of rice. With the RILs, the QTLs identified under controlled environment condition can be tested in the field, because seeds can easily be produced for use in replicated experiments.

\section{ACKNOWLEDGEMENTS}

This work was supported by a grant from the NextGeneration Biogreen 21 Program (Plant Molecular Breeding Center No. PJ008136), Rural Development Administration, Republic of Korea.

\section{REFERENCES}

Abdelkhalik AF, Shishido R, Nomura K and Ikehashi $\mathrm{H}$. 2005. QTL-based analysis of leaf senescence in an indica/japonica hybrid in rice (Oryza sativa L.). Theor. Appl. Genet. 110:1226-1235.
Andaya VC and Mackill DJ. 2003. Mapping of QTLs associated with cold tolerance during the vegetative stage in rice. J. Expt. Bot. 54:2579-2585.

Andaya VC and Tai TH. 2006. Fine mapping of the qCTS12 locus, a major QTL for seedling cold tolerance in rice. Theor. Appl. Genet. 113:467-475.

Andaya VC and Tai TH. 2007. Fine mapping of the qCTS4 locus associated with seedling cold tolerance in rice (Oryza sativa L.). Mol. Breed. 20:349-358.

Causse MA, Fulton TM, Cho YG, Ahn SN, Chunwongse J, Wu K, Xiao J, Yu Z, Ronald PC, Harrington SE, Second G, McCouch SR and Tanksley SD. 1994. Saturated molecular map of the rice genome based on an interspecific backcross population. Genetics 138:1251-1274.

IRRI. 1979. Report of a rice cold tolerance workshop. In: IRRI Proc. Rice Cold Tolerance Workshop, Office of Rural Development, Suweon, Korea, p.139.

Ishimaru K, Yano M, Aoki N, Ono K, Hirose T, Lin SY, Monna L, Sasaki T and Ohsugi R. 2001. Toward the mapping of physiological and agronomic characters on a rice function map: QTL analysis and comparison between QTLs and expressed sequence tags. Theor. Appl. Genet. 102:793-800.

Jeong EG, Yea JD, Baek MK, Moon HP, Choi HC, Yoon KM and Ahn SN. 2000. Estimation of critical temperature for traits related to cold tolerance in rice. Korean J. Breed. 32:363-368.

Jeoung SW, Choi SM, Lee DS, Ahn SN, Hur YK, Chow WS and Park YI. 2002. Differential susceptibility of photosynthesis to light chilling stress in rice (Oryza sativa L.) depends in the capacity for photochemical dissipation of light. Mol. Cells. 13:419-428.

Ji ZZ, Zeng YX, Zeng DL, Ma LY, Li XM, Liu BX and Yang CD. 2010. QTL for rice cold tolerance identified at plumule and 3-leaf-seedling stage using QTL network software. Rice Sci. 17:282-287.

Kim DS, Yoon YH, Shin JC, Kim JK and Kim SD. 2002. Varietal difference in relationship between SPAD value and chlorophyll and nitrogen concentration in rice leaf. Korean J Crop Sci. 47:263-267.

Kura-Hotta M, Satoh K and Katoh S. 1987. Relationship between photosynthesis and chlorophyll content during leaf senescence. Plant Cell Physiol. 28:1321-1329.

Misawa S, Mori N, Takumi S, Yoshida S and Nakamura C. 2000. Mapping of QTLs for low-temperature response in seedlings of rice (Oryza sativa L.). Cereal Research Communications 28:33-40. 
McCouch SR and Doerge RW. 1995. QTL mapping in rice. Trends Genet. 11:482-487.

McCouch SR, Teytelman L, Xu Y, Lobos KB, Clare K, Walton M, Fu B, Maghirang R, Li Z, Xing Y, Zhang Q, Kono I, Yano M, Fjellstrom R, Declerck G, Schneider D, Cartinhour S, Ware D and Stein L. 2002. Development and mapping of 2240 new SSR markers of rice (Oryza sativa L.). DNA Res. 9:199-207.

Oh CS, Choi YH, Lee SJ, Yoon DB, Moon HP and Ahn SN. 2004. Mapping of quantitative trait loci for cold tolerance in weedy rice. Breeding Sci. 54:373-380.

Oh CS, Park IK, Kim DM and Ahn SN. 2010. QTL mapping of agronomic traits using an introgression line population derived from an intersubspecific cross in rice. Korean J. Breed. Sci. 42:470-480.

Panaud O, Chen X and McCouch SR. 1996. Development of microsatellite markers and characterization of simple sequence length polymorphism (SSLP) in rice (Oryza sativa L.). Mol. Gen. Genet. 252:597-607.

Saito K, Hayano-Saito Y, Maruyama-Funatsuki W, Sato Y and Kato A. 2004. Physical mapping and putative candidate gene identification of a quantitative trait locus $\mathrm{Ctb} 1$ for cold tolerance at the booting stage of rice. Theor. Appl. Genet. 109:515-522.

Suh JP, Ahn SN, Choi IS, Cho YC, Hwang HG and Suh HS. 2003. Identification of QTLs for cold tolerance at seedling stage in korean weedy rice (Oryza Sativa L.). Korean J. Breed. 35:96-101.

Suh JP, Jeung JU, Lee JI, Choi YH, Yea JD, Virk PS, Mackill DJ and Jena KK. 2010. Identification and analysis of QTLs controlling cold tolerance at the reproductive stage and validation of effective QTLs in cold-tolerance genotypes of rice (Oryza sativa L.). Theor. Appl. Genet. 120:985-995.

Takai T, Kondo M, Yano M and Yamamoto T. 2010. A quantitative trait locus for chlorophyll content and its association with leaf photosynthesis in rice. 3:172-180.

Wang S, Basten CJ and Zeng ZB. 2007. Windows QTL Cartographer 2.5. Department of Statistics, North Carolina State University, Raleigh, NC. http://statgen.ncsu.edu/ qtlcart/WQTLCart.htm

Watanabe SY, Hatanaka Y and Inada K. 1980. Development of a digital chlorophyll meter: I. Structure and performance. Japanese J. Crop Sci. 49 (special issue):89.

Yoshida R, Kanno A, Sato T and Kameya T. 1996. Cool temperature induced chlorosis in rice plants. Plant Physiol. 110:997-1005.

Zhang ZH, Li S, Li W, Chen W and Zhu YG. 2005. A major QTL conferring cold tolerance at the early seedling stageusing recombinant inbred lines of rice (Oryza sativa L.). Plant Sci. 168:527-534. 